\title{
Variability of Southern Ocean Jets near Topography
}

\author{
CHRISTOPHER C. CHAPMAN \\ Research School of Earth Sciences, and ARC Centre of Excellence for Climate System Science, Australian National \\ University, Canberra, Australian Capital Territory, and CSIRO Wealth From Oceans Flagship, Hobart, Tasmania, Australia \\ ROSEMARY MORROW \\ Laboratoire d'Etudes en Géophysique et Océanographie Spatiales, Toulouse, France
}

(Manuscript received 1 August 2013, in final form 31 October 2013)

\begin{abstract}
The interaction of jets with topography in the Southern Ocean is investigated using 19 years of altimetry data. In particular, the "jet jumping" mode of variability, by which two or more jets passing close to the same topographic feature show strongly anticorrelated strengthening and weakening, is studied. Three regional case studies are described-the Southeast Indian Ridge south of Tasmania, the Macquarie Ridge south of New Zealand, and the Pacific-Antarctic Rise-where the jet jumping variability is found to occur. Using principal component analysis, the spatial patterns of variability show a vortex dipole forming on either side of a particular jet. For each regional study, it is found that the variability in strength of these vortices (as measured by the spatially averaged vorticity) is strongly correlated with time series of the principle component that describes the jet jumping variability. The observational analysis is complemented by a suite of idealized numerical experiments using a three-layer quasigeostrophic model with simple topography. The numerical results show similar spatial patterns of variability to those observed in the altimetric data. Internal variability is sufficient to generate jet jumping variability, as there is no time-varying external forcing applied in the model configuration. The simulations are used to investigate the effect of topographic scale and changing bottom friction. The authors find that both have a strong influence on the time scale of the variability, with larger topographic scales and higher bottom friction leading to faster time scales. This study shows that even in regions where the flow is strongly influenced by topography, Southern Ocean jet flow may exhibit low-frequency variability.
\end{abstract}

\section{Introduction}

The circulation in the Southern Ocean is dominated by the Antarctic Circumpolar Current (ACC), which is composed of a series of strong, narrow eastward currents known as jets (Rintoul et al. 2001). Jets are a common feature in geophysical fluids having been found in the midlatitude troposphere and stratosphere, in the atmospheres of gas giant planets, and numerous laboratory flows (Thompson 2008). They consist of large-scale, predominantly zonal flow that persists with time and, unlike the jets of classical fluid mechanics, are not caused by input momentum condition, but arise spontaneously from interaction of turbulence, mean

Corresponding author address: C. C. Chapman, Research School of Earth Sciences, The Australian National University, Building 142, Mills Road, Canberra, ACT 0200, Australia.

E-mail: chris.chapman@anu.edu.au flow, and the background potential vorticity gradient (Rhines 1994). Within the Southern Ocean, jets are related to the hydrographic fronts that have been the subject of many studies over the preceding several decades (Sokolov and Rintoul 2007). Topography exerts a strong influence over Southern Ocean jets, influencing their location (Graham et al. 2012; Sokolov and Rintoul 2009), orientation (Thompson 2010), the formation of turbulent eddies (Williams et al. 2007; Hughes and Ash 2001), and the transfer of momentum from the surface through to the abyssal depths and into the earth's surface through interfacial form stress (Ward and Hogg 2011).

The availability of high-quality remotely sensed datasets, improved networks of in situ data from autonomous drifters, and the continuing advances in numerical modeling have enabled us to study the time variability of these jets. This is in contrast to earlier studies that primarily made use of hydrographic data, which gave a more steady-state view of the Southern Ocean (e.g., Orsi et al. 


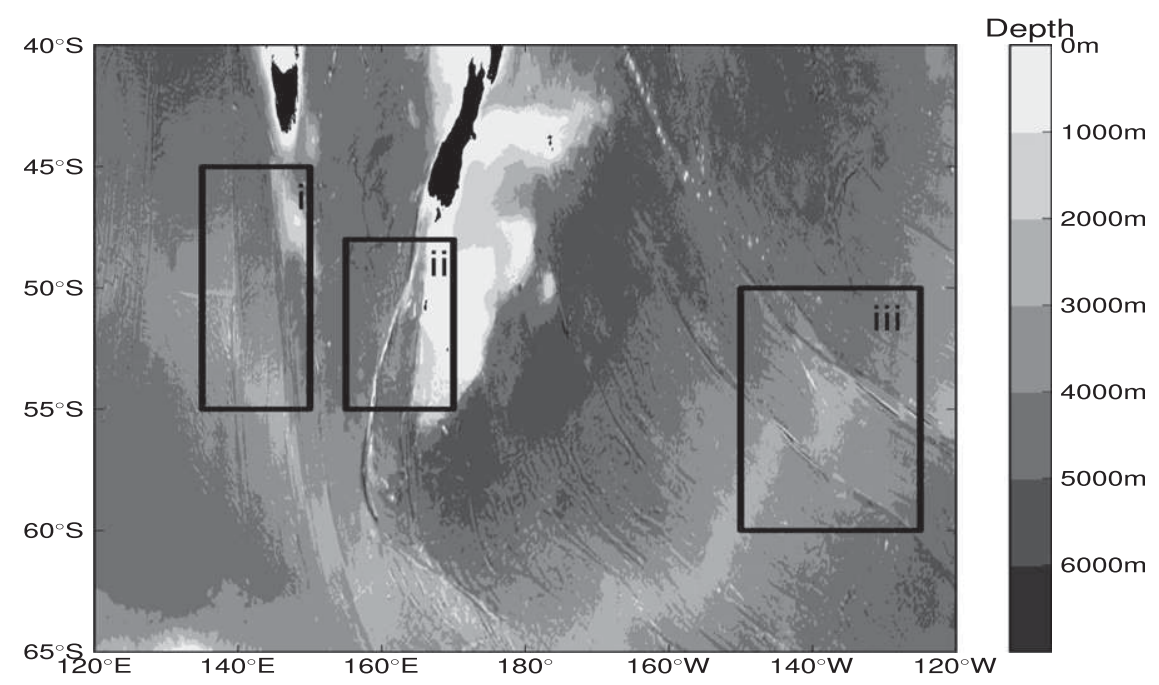

FIG. 1. Southern Ocean depth between $120^{\circ} \mathrm{E}$ and $120^{\circ} \mathrm{W}$ taken from the ETOPO1 dataset, showing major bathymetric features. Boxes denote the three study regions: (i) Southeast Indian Rise, (ii) Macquarie Ridge, and (iii) Pacific-Antarctic Ridge.

1995). Such time-dependent studies have revealed the circulation of the Southern Ocean to exhibit variability on a variety of spatial and temporal scales (Sokolov and Rintoul 2009; Thompson and Richards 2011).

In this paper, we investigate a mode of low-frequency variability described as "jet jumping" (Chapman and Hogg 2013) using a hybrid observation-modeling approach. Evidence of this phenomena was first found in a network of fixed moorings placed to measure the transport associated with a jet of the ACC that passes through gaps (or "canyons") in the Macquarie Ridge, a large topographic feature south of New Zealand (Rintoul et al. 2013, manuscript submitted to Nat. Geosci.). The one year of data obtained from the moorings revealed that the current through each canyon exhibited substantial temporal variability. Furthermore, the transport through two adjacent canyons was highly anticorrelated. During periods of anomalously high transport through the northern canyon, transports through the adjacent, southern canyon were typically anomalously low. Hydrographic sections obtained from repeat CTD sections during the same experiment suggested that the position of the hydrographic fronts changed only rarely or was ambiguous.

A dynamical explanation of these phenomena was presented in Chapman and Hogg (2013), who used an idealized, eddy-resolving quasigeostrophic numerical model to simulate the jet jumping behavior. The authors related the variability of the strength of the jets to the strength of vortices that form between the canyons in regions where the topography forms closed geostrophic contours. With the success of the dynamical framework in explaining the jet jumping variability in the idealized numerical model, an obvious question is whether this framework translates to the ocean.

Three regions are chosen for study, shown in Fig. 1: the Southeast Indian Rise (Fig. 1, region i), the Macquarie Ridge (region ii), and the Pacific-Antarctic Ridge (region iii). Common to each region is a meridionally orientated topographic feature that is traversed by two or more jets. Two of these three study regions (the Southeast Indian Rise and the Pacific-Antarctic Ridge) coincide with those of Thompson and Richards (2011), which showed substantial variability of the current in these regions. Unlike that study, however, here we focus solely on the jet jumping mode of variability.

\section{Dynamics of jet jumping}

In this section, we briefly discuss the mechanism that leads to the jet jumping mode of variability. The reader is referred to Chapman and Hogg (2013) for details.

Imagine two quasi-zonal eastward-flowing jets separated by some meridional distance $\Delta Y$, shown schematically in Fig. 2, in an ocean of unperturbed depth $H$. The two jets interact with a meridional ridge with two zonally oriented canyons (gaps in the ridge) separated by a distance, roughly, $\Delta Y$. Owing to potential vorticity conservation, as the ridge shoals the ocean, the jets are steered equatorward upstream and poleward downstream of the ridge. The interaction of the ridge with the background flow can, in certain circumstances, generate a vortex [or sometimes a vortex dipole, as was the case in Chapman and Hogg (2013)] on either side of the canyons. There are numerous mechanisms to generate these vortices. In 


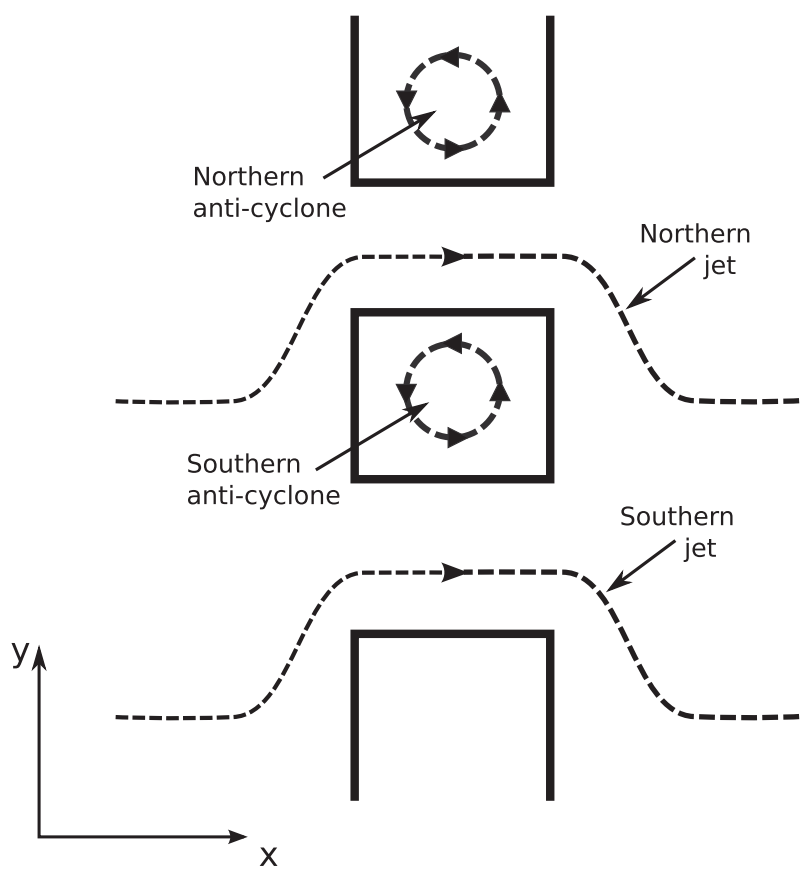

FIG. 2. Schematic showing two quasi-zonal jets interacting with two topographically generated vortices; a situation that can give rise to the jet jumping mode of variability.

Chapman and Hogg, they were generated by the interaction of turbulent flow components and closed geostrophic contours, as in Dewar (1998). They could also be produced by standing Rossby waves (Völker 1999) or trapped Kelvin waves (Marshall 2011). Vortices are, to first order, barotropic, while jets are baroclinic (although equivalent barotropic).

Once formed, the barotropic vortices wax and wane in strength and interact with the baroclinic jet flow to cause the anticorrelated variations in jet transport referred to as jet jumping. Previous work has shown that vortices interacting with jets have the ability to change both the strength and position of that jet (Stern and Flierl 1987). In the situation described in Fig. 2, if the southern vortex were to increase in anticyclonicity, this would act to reinforce the southern jet and decrease the transport of the northern jet. Were the southern vortex to decrease in anticyclonicity, the reverse situation would apply and the northern jet would increase in strength at the expense of the southern jet.

Based on this mechanism, there is a relationship between the variability in strength of the topographic vortices and the strength of the jets passing near them. The numerical simulations of Chapman and Hogg (2013) showed this relationship manifests as a strong correlation between the transport carried by a particular jet and a nearby vortex. This relationship will be explored in the Southern Ocean using satellite altimetry data in section 4 .
The internal dynamics controlling the jet jumping will be further explained using the numerical model of Chapman and Hogg (2013) in section 5 with idealized configurations reminiscent of the observed cases from altimetry.

We note that the mechanism described by Chapman and Hogg (2013) does not necessarily imply that jets are shifting their mean positions. Instead, this mechanism implies that two neighboring jets with fixed locations are undergoing anticorrelated strengthening/weakening. This interpretation is supported by fixed mooring measurements of the flow through gaps in the Macquarie Ridge (Rintoul et al. 2013, manuscript submitted to Nat. Geosci.) that show anticorrelated fluctuations through adjacent gaps. However, the repeat CTD sections indicate that fronts (at least as defined by hydrographic variables such as sharp temperature or salinity changes) only rarely shift their position, despite the dramatic variation in transport through the gaps.

\section{Data and methodology}

\section{a. Bathymetry}

To describe the subsurface topography, we use data from the 1 arc-minute global relief model of Earth's surface (ETOPO1) project (Amante and Eakins 2009). Data are projected on a standard latitude-longitude grid with a grid spacing of 1 arc-min.

\section{b. Time-mean dynamic topography}

To estimate the mean state of the ocean we use the combined mean dynamic topography (CMDT) product (Rio et al. 2011) (http://www.aviso.oceanobs.com/en/ data/products/auxiliary-products/mdt/). The mean sea surface is reconstructed over the period 1993-99 by combining data from the Gravity Recovery and Climate Experiment (GRACE) mission, satellite altimeters, and drifting buoys. Data were interpolated from the $1 / 30^{\circ}$ latitude-longitude grid to a $1 / 3^{\circ}$ Mercator grid to enable comparison with the time-varying gridded altimetric sea level anomaly data.

\section{c. Time-varying dynamic topography}

The satellite dataset used in this study is the Archiving, Validation, and Interpretation of Satellite Oceanographic data (AVISO) weekly gridded sea level anomalies (SLA) (http://www.aviso.oceanobs.com/en/data/products/seasurface-height-products.html). We use dynamic topography from the 19-yr period 1993-2012, giving exactly 1000 data records. These data are mapped to a $1 / 3^{\circ}$ Mercator grid using optimal interpolation of alongtrack data series based on the REF dataset, which uses two satellite missions [Ocean Topography Experiment 
(TOPEX)/Poseidon/European Remote Sensing Satellite (ERS) or Jason-1/Envisat or Jason-2/Envisat] with consistent sampling over the 19-yr period. Data are corrected for instrumental errors, atmospheric perturbations, orbit errors, tides, inverted barometer bias, and aliased fast barotropic signals (periods of less than 20 days). Details of the processing and mapping technique are given in Dibarboure et al. (2011).

In the following study, we use "absolute dynamic topography," which is the sum of the time-mean dynamic topography and time-varying sea level anomalies.

\section{d. Diagnostics}

The diagnostics used in this paper are similar to those used in Chapman and Hogg (2013). In particular, we diagnose the

(i) temporal variability of jet strength,

(ii) spatial patterns of sea surface height ( $\mathrm{SSH}$ ) associated with jet strength variability, and

(iii) temporal variability of topographic vortices.

\section{1) TEMPORAL VARIABILITY OF JET STRENGTH}

The net transport of a particular jet pathway is used here as a metric for the current strength. Assuming geostrophic balance, the transport perpendicular to a line $L$ with endpoints $L_{1}$ and $L_{2}$ and unit normal $\mathbf{n}$ is given by

$$
T=\frac{g H}{f} \int_{L} \mathbf{e}_{z} \times \nabla \eta \cdot \mathbf{n} d l=\frac{g}{f}\left(\eta_{L_{2}}-\eta_{L_{1}}\right),
$$

where $\eta$ is the absolute dynamic topography, $g$ the gravitational acceleration, $H$ the fluid depth, and $f$ the Coriolis parameter; we have assumed that the variation of $f$ is small. Transport time series are low-pass filtered with a windowed-sinc filter (using a Blackman window) designed to compromise between time and frequency domain performance (Smith 2003, p. 285). The end points $L_{1}$ and $L_{2}$ will be indicated in each case study. We choose a cutoff period of 90 days, which is a typical eddy time scale in the Southern Ocean (Phillips 2000, p. 67).

\section{2) Spatial PATterns of SSH}

As in Chapman and Hogg (2013) and Berloff et al. (2007), we use "key state analysis" to determine the spatial SSH structure associated with the jet jumping mode of variability. However, the procedure is modified slightly in order to improve the representation of the lowfrequency mode and reduce the influence of noise and other variability. To do this, we compute the transport through two adjacent regions [or canyons as in Chapman and Hogg (2013)], described for each case study in sections 4 and 5. Taken together, these time series constitute a two-dimensional dynamical system. A standard principal component analysis (PCA) of a two-dimensional time series will result in two principal components (PCs): one that describes correlated behavior and the other that describes anticorrelated behavior (Wilks 2006). We use the anticorrelated PC to describe the jet jumping behavior.

Then, we form conditional ensemble averages of SSH using the PC time series associated with the jet jumping variability. The system is defined as existing in state " $\mathrm{A}$ " when the PC time series is one standard deviation or greater than the mean. Likewise, we define state " $B$ " as when the PC time series is one standard deviation or less than the mean. Then, we form composites of all time steps when the system exists in either key state by averaging them to form the ensemble mean.

\section{3) TEMPORAL VARIABILITY OF TOPOGRAPHIC VORTICES}

In their investigation of the variability of the Zapiola anticyclone, Volkov and $\mathrm{Fu}$ (2008) found that they could adequately describe the time-varying behavior using spatially averaged vorticity obtained from satellite altimetry. Here, we follow this approach. The spatially averaged vorticity was computed as

$$
\langle\zeta\rangle_{\Omega}=\int_{\Omega}\left(\frac{g}{f}\right) \nabla^{2} \eta d A
$$

The integration domain $\Omega$ is determined by investigating the spatial patterns of variability described in step (ii) above and will be indicated in each case study. We take special care in the computation of the Laplacian term in Eq. (2). To avoid vorticity-dependent bias associated with narrow finite difference stencils, we use a larger 7-point finite difference stencil (Arbic et al. 2012). All computations are carried out in a Mercator coordinate system in order to implicitly include the latitudinal variation in grid spacing (see the appendix).

\section{Jet jumping variability in the Southern Ocean}

In this section, we describe the jet jumping variability in three different regions as described in section 2. Each region contains localized jet topography interactions. The data for each case study are taken from the Rio et al. (2011) and AVISO sea surface height datasets described in sections $2 b$ and $2 c$.

\section{a. Southeast Indian Ridge}

The Southeast Indian Ridge (here between $135^{\circ}$ and $145^{\circ} \mathrm{E}$ to the south and west of Tasmania) is a diagonally oriented topographic feature (Fig. 3). The region of interest here is located at approximately $53^{\circ} \mathrm{S}, 140^{\circ} \mathrm{E}$ and 

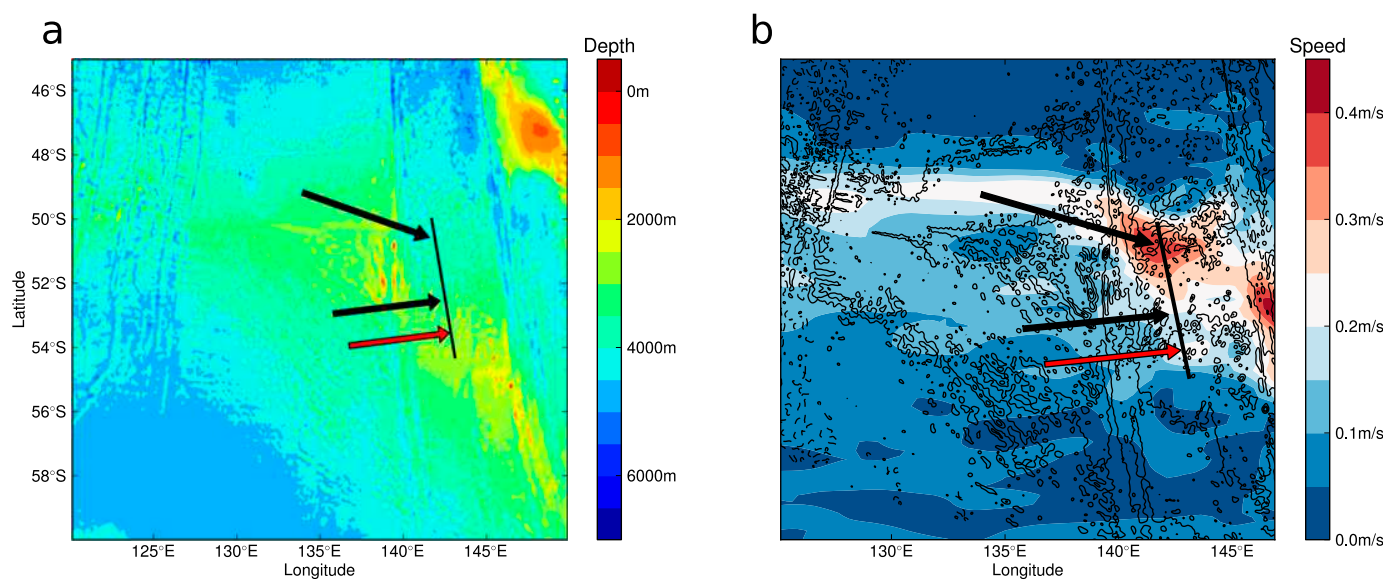

FIG. 3. Southeast Indian Ridge (a) bathymetry and (b) mean flow speed. Black arrows indicate the positions of jet cores involved in the jet jumping mode, and the red arrow indicates the position of the stable jet. The transect line for Fig. 4 is also shown.

consists of a plateau rising steeply to the south. The topographic slope acts to change the local background potential vorticity gradient and influences the positions of jets in the vicinity. The region is known to be traversed by jets associated with the Subantarctic Front and the Polar Front (Sokolov and Rintoul 2007). The mean current speed [from the Rio et al. (2011) dataset] indicates two eastward-flowing currents, shown in Figs. 4a and $4 \mathrm{c}$. These currents are found at approximately $53.5^{\circ}$ and $50.5^{\circ} \mathrm{S}$, labeled with arrows in Fig. 3. There is also a third, highly variable, current that flows between those two with an approximate center at $52.3^{\circ} \mathrm{S}$, also labeled with an arrow.

A transect of mean zonal velocity is taken along the line shown in Fig. 3 and displayed in Fig. 4a. The three currents indicated with arrows in Fig. 3 are labeled (from north to south) 1,2 , and 3 . The mean transport of the northern current is computed using Eq. (1) with limits $L_{1}=\left(51.5^{\circ} \mathrm{S}, 142.1^{\circ} \mathrm{E}\right)$ and $L_{2}=\left(50.0^{\circ} \mathrm{S}, 141.7^{\circ} \mathrm{E}\right)$ to be $27.5 \mathrm{~Sv}\left(1 \mathrm{~Sv} \equiv 10^{6} \mathrm{~m}^{3} \mathrm{~s}^{-1}\right)$, substantially higher than the mean transport of the southern current $\left[L_{1}=\left(54.0^{\circ} \mathrm{S}\right.\right.$, $\left.143.3^{\circ} \mathrm{E}\right)$ and $\left.L_{2}=\left(53.0^{\circ} \mathrm{S}, 142.8^{\circ} \mathrm{E}\right)\right]$ at $18.0 \mathrm{~Sv}$. The mean transport of the center current is $21.3 \mathrm{~Sv}\left[L_{1}=\right.$ $\left(53.5^{\circ} \mathrm{S}, 143.1^{\circ} \mathrm{E}\right)$ and $\left.L_{2}=\left(52.2^{\circ} \mathrm{S}, 142.6^{\circ} \mathrm{E}\right)\right]$.

We compute the transport time series of these three currents and find that the northern and southern current (currents 1 and 3) transport time series are slightly correlated, with PCA showing that $\sim 48 \%$ of the variance is explained by the anticorrelated mode. In contrast, the northern and center currents (currents 1 and 2) are substantially anticorrelated (anticorrelation explaining $\sim 66 \%$ of the variance). The key state analysis, shown as a transect of zonal velocity in Fig. $4 \mathrm{~b}$, illustrates the anticorrelated strengthening/weakening of the jets 1 and 2. State A corresponds to a stronger center jet and a weaker northern jet, with the reverse situation occurring in key state B. This anticorrelated strengthening and weakening is characteristic of the jet jumping mode of variability. The southernmost current (current 3 ) is not strongly affected by the jet jumping variability at the Southeast Indian Ridge.

The variability of the flow in this region is shown in the Hovmöller diagram in Fig. 5a. In contrast to the variability near the Macquarie Ridge (described in section 4b) meridional shifts of the jet from the north to the south can be seen. The transport of jets 1 and 2 (shown as the black lines in Fig. 5a) are computed and shown in

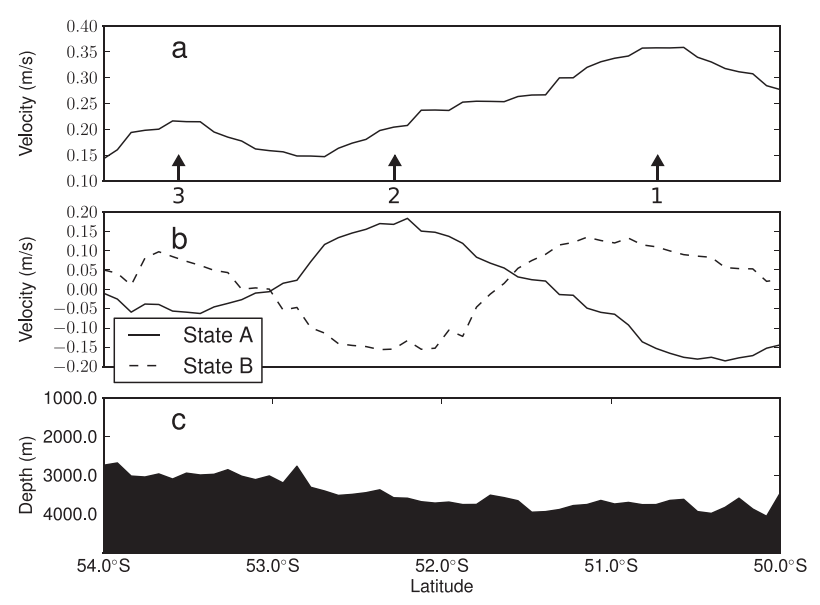

FIG. 4. Transect along the solid line in Fig. 3 showing (a) mean zonal velocity. Arrows indicate the approximate jet core locations. The jets labeled 1 and 2 undergo jet jumping, while the jet labeled 3 does not. (b) Anomalous zonal velocity in key state A (solid) and key state B (dashed) and (c) bathymetry. 

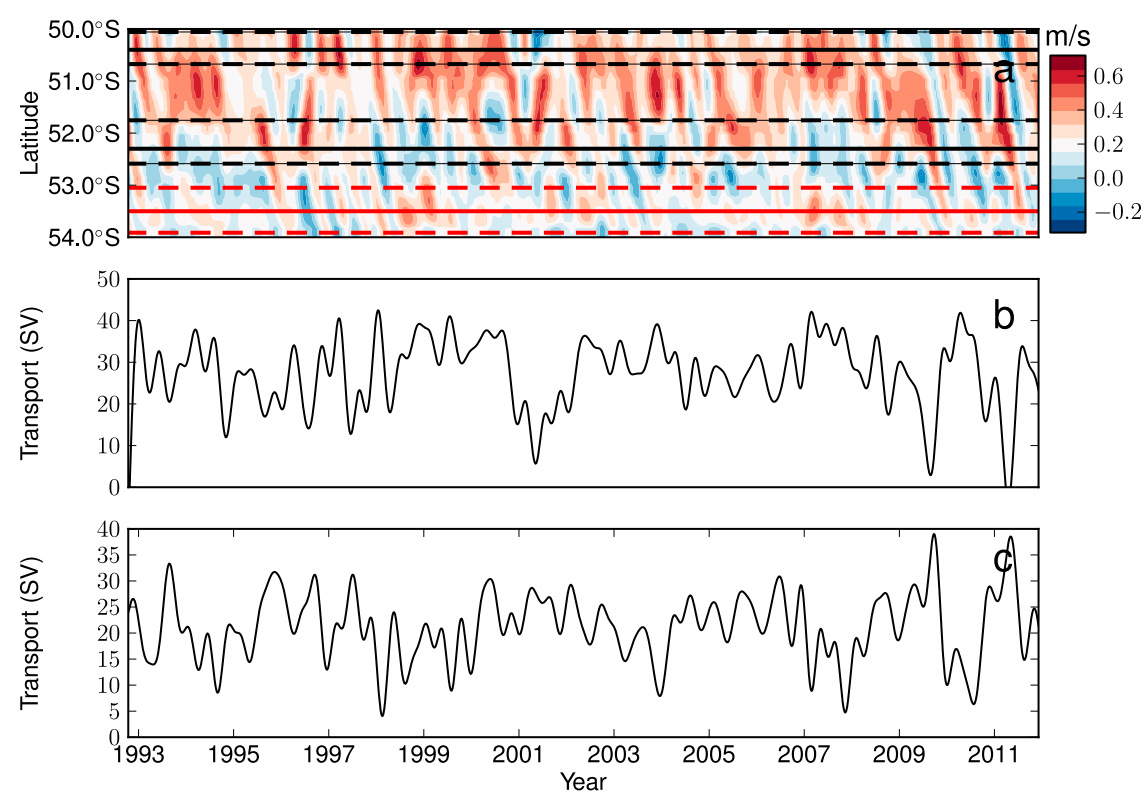

FIG. 5. Temporal variability of the Southern Ocean near the Southeast Indian Ridge: (a) latitude-time Hovmöller diagram of zonal velocity. The solid black lines indicate locations of jet cores involved in jet jumping. The thick red line indicates the location of the stable jet. Dashed lines indicate the northern and southern extents used in the transport computation:

(b) northern jet transport and (c) central jet transport.

Figs. 5b,c. The transport is highly variable, varying between -2 and $41 \mathrm{~Sv}$ in the northern position (where negative values indicate westward, or reversed, flow) and 5 and $39 \mathrm{~Sv}$ in the center position. The time scale of the variability is determined by spectral analysis of the PC time series associated with the jet jumping (i.e., the anticorrelated) mode. The dominant period is found to be approximately $2.6 \mathrm{yr}$, however, at low frequencies, although the power in the spectrum is distributed between 1.3 and $3.2 \mathrm{yr}$.

\section{b. Macquarie Ridge}

The Macquarie Ridge topography (Fig. 6a) consists of a meridionally oriented ridge, located to the west of a large feature called the Campbell Plateau. In the particular region of interest, the ridge contains two gaps or canyons, which are preferred pathways for at least one ACC jet associated with the Subantarctic Front at $52^{\circ}$ and $53.5^{\circ} \mathrm{S}$. The mean current speed in this region is shown in Fig. 6b. As with the Southeast Indian Rise,
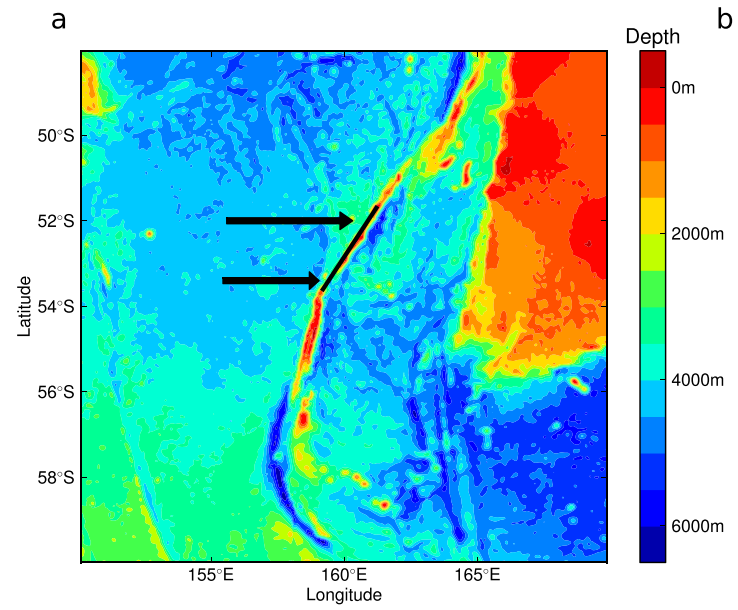

b

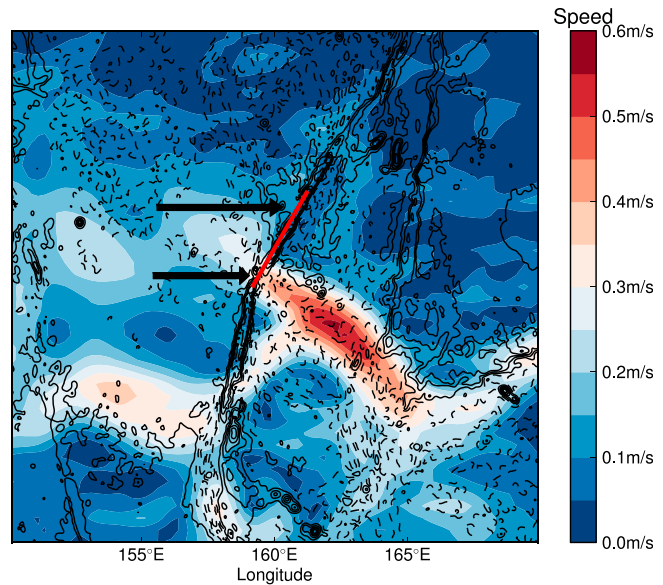

FIG. 6. Macquarie Ridge (a) bathymetry and (b) mean flow speed. Arrows indicate the positions of canyons. The transect line for Fig. 7 is also shown. 
transport is computed through the northern and southern canyons using Eq. (1). The limits are taken to be $L_{1}=\left(53.6^{\circ} \mathrm{S}, 159.2^{\circ} \mathrm{E}\right)$ and $L_{2}=\left(53.0^{\circ} \mathrm{S}, 159.9^{\circ} \mathrm{E}\right)$ for the southern canyon and $L_{1}=\left(52.4^{\circ} \mathrm{S}, 160.6^{\circ} \mathrm{E}\right)$ and $L_{2}=$ $\left(51.7^{\circ} \mathrm{S}, 161.2^{\circ} \mathrm{E}\right)$ for the northern canyon. We find that the majority of the current flows through the southern canyon with a mean transport of $39.2 \mathrm{~Sv}$, which dominates the mean transport through the northern canyon (1.9 Sv). The transport values computed from the altimetry data are checked and calibrated against the transports computed from fixed moorings described in Rintoul et al. (2013, manuscript submitted to Nat. Geosci.). We find that the satellite altimeter-derived transports agree well with the fixed mooring data during the period when both sets of observations are available.

A transect of the time-mean zonal current along the line in Fig. 6 is shown in Fig. 7a, together with the bathymetry (Fig. 7c). Here, we see clearly that the southern jet is aligned with the southern canyon. After computing transport through both canyons, we perform the key state analysis (Fig. 7b). Key state A shows a stronger jet through the southern canyon, with an average peak velocity anomaly of about $10 \mathrm{~cm} \mathrm{~s}^{-1}$, while key state B shows a stronger jet in the northern canyon. The anticorrelated mode explains approximately $60 \%$ of the variance, indicating strong anticorrelation.

The variability in transport through the north and south canyons over the satellite period is shown in Fig. 8 . We note substantial variability in the transport, with
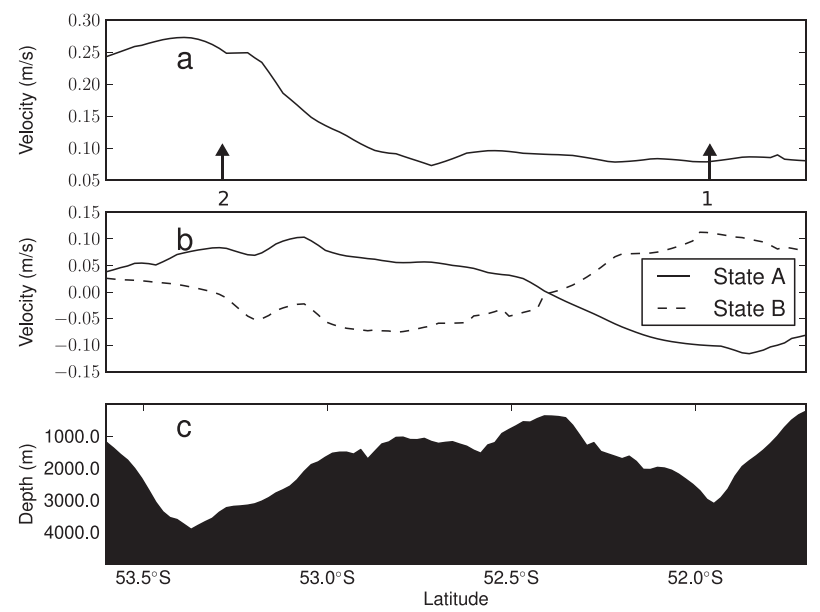

FIG. 7. Transect along the solid line in Fig. 6 showing (a) mean zonal velocity (arrows indicate the approximate jet core locations), (b) anomalous zonal velocity in key state A (solid) and key state B (dashed), and (c) bathymetry.

transport in the dominant southern canyon varying between 31 and $50 \mathrm{~Sv}$, while flow in the northern canyon varies between -6 and $17 \mathrm{~Sv}$. Unlike at the Southeast Indian Ridge, the Hovmöller diagram shows little evidence of meridional jet movement between the two canyons. However, we can see in Fig. $7 \mathrm{~b}$ that the southern jet undergoes northward displacement by around $0.25^{\circ}$ when it is in either key state. This indicates that the jets are strongly bound to the canyon regions. This is in
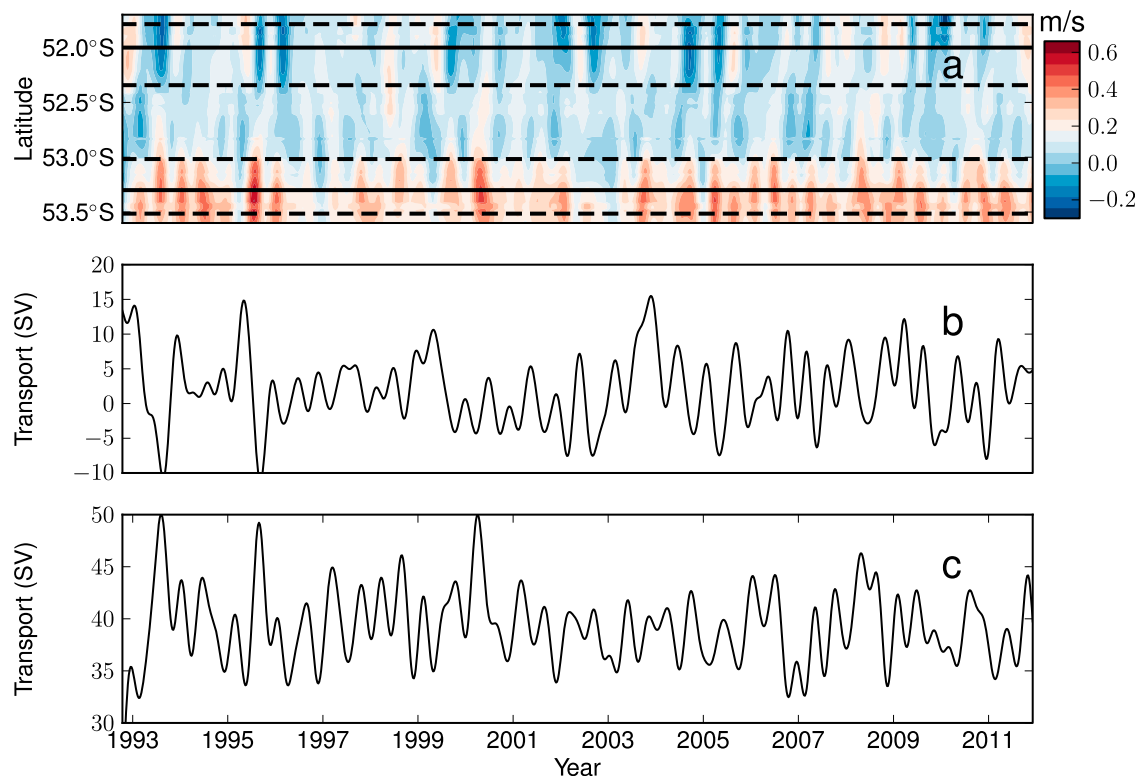

FIG. 8. Variability of the Southern Ocean in the vicinity of the Macquarie Ridge: (a) latitudetime Hovmöller diagram of zonal velocity (thick black lines indicate approximate canyon center latitudes. Dashed lines indicate the northern and southern extents used in the transport computation). (b) Transport through (b) the northern canyon and (c) the southern canyon. 

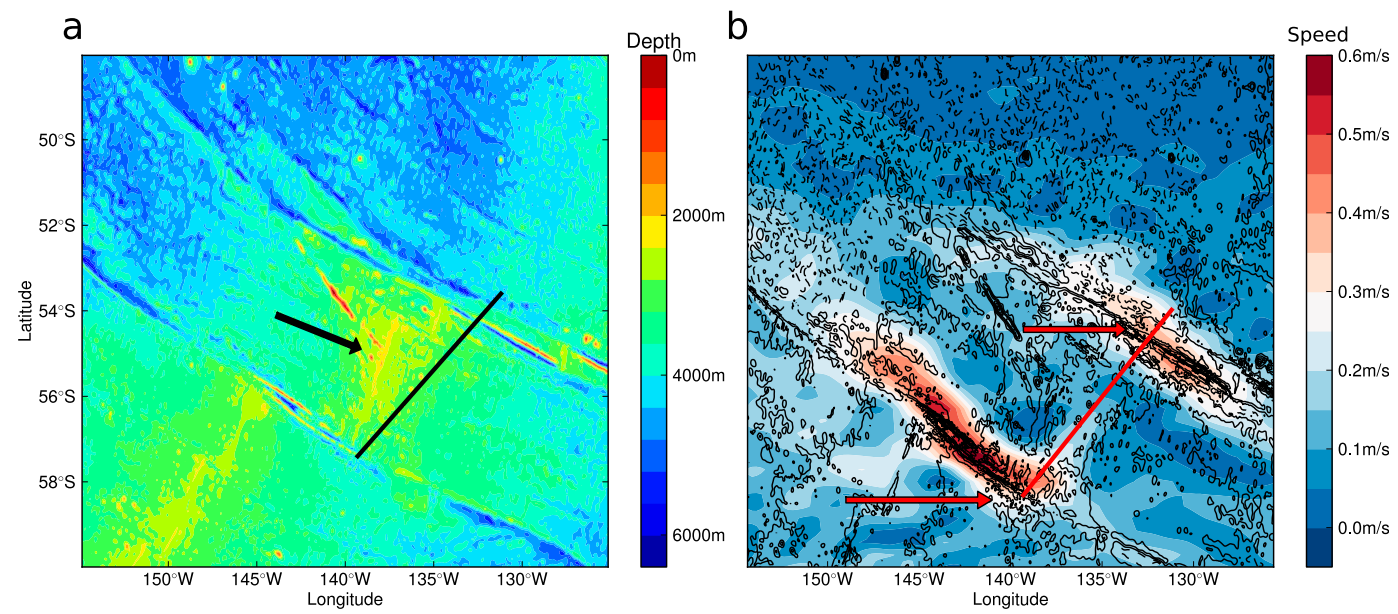

FIG. 9. Pacific-Antarctic Rise (a) bathymetry (arrow indicates the positions of the ridge discussed in text) and (b) mean flow speed (arrows indicate the location of the jets). The transect line for Fig. 11 is also shown.

contrast to the situation at the Southeast Indian Ridge, where there is stronger evidence of shifting jet positions. Here, the northern jet instead exhibits current reversals and westward flows when the southern jet is strong.

The time scale of the jet jumping mode is determined through spectral analysis of the PC time series, which shows the dominant period to be approximately $2.21 \mathrm{yr}$. The spectrum is not as broad as at the Southeast Indian Ridge or the Pacific-Antarctic Rise.

\section{c. Pacific-Antarctic Rise}

The Pacific-Antarctic Rise topography, shown in Fig. 9a, consists of a complex northeast-southwest aligned midarea ridge at $55^{\circ} \mathrm{S}, 138^{\circ} \mathrm{W}$. This ridge is flanked to the north and south by northwest-southeastoriented fracture zones. The mean current speed (Fig. 9b) shows two jets lying north and south of this sharp displaced ridge, aligned with the northwest-southeast fracture zones.

The transect of zonal velocity (Fig. 10a) clearly shows two jet cores located at $57^{\circ}$ and $54^{\circ} \mathrm{S}$. The southern jet, with a mean speed of $\sim 45 \mathrm{~cm} \mathrm{~s}^{-1}$ is stronger than the northern jet, which has a mean speed of $\sim 35 \mathrm{~cm} \mathrm{~s}^{-1}$. Despite the higher peak velocity, the southern jet carries substantially less transport than the northern jet $(20.4 \mathrm{~Sv}$ for the southern jet, compared to $30.2 \mathrm{~Sv}$ for the northern jet) as the northern jet is both wider and the water column deeper. The key state analysis (Fig. 10b) shows that, when the system exists in key state A, the northern jet is weaker, with negative peak velocity anomalies of $\sim 15 \mathrm{~cm} \mathrm{~s}^{-1}$, while the southern jet is stronger by $\sim 5 \mathrm{~cm} \mathrm{~s}^{-1}$. Key state B shows an approximate mirror situation. The anticorrelated jet jumping mode explains $\sim 55 \%$ of the variance.
The strength of the two jets is highly variable. The Hovmöller diagram in Fig. 11a shows the northern and southern jet strengthening and weakening on a time scale of $3.2 \mathrm{yr}$. Both jets show evidence of occasional meridional propagation (e.g., in early 2001 and early 2004). Northern jet transport (Fig. 11b) varies between 0 and $50 \mathrm{~Sv}$, while the southern canyon transport varies between 2 and 34 Sv (Fig. 11c).

\section{d. Spatial structure of jet jumping variability}

For each case study, the spatial pattern of the altimetric SSH associated with the jet jumping mode of the variability is determined using the key state analysis described in section $3 \mathrm{~d}(2)$.
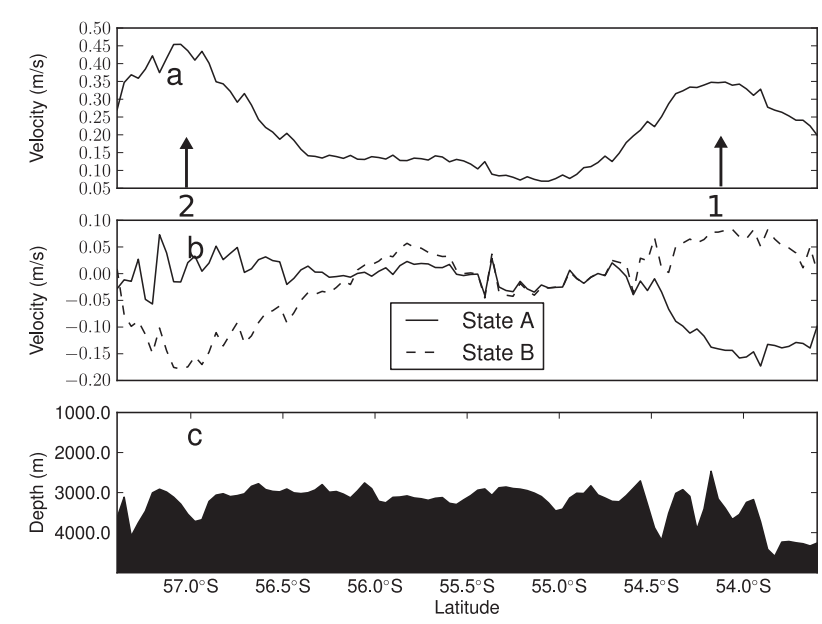

FIG. 10. Transect along the solid line in Fig. 9 showing (a) mean zonal velocity (arrows indicate the approximate jet core locations), (b) anomalous zonal velocity in key state A (solid) and key state B (dashed), and (c) bathymetry. 

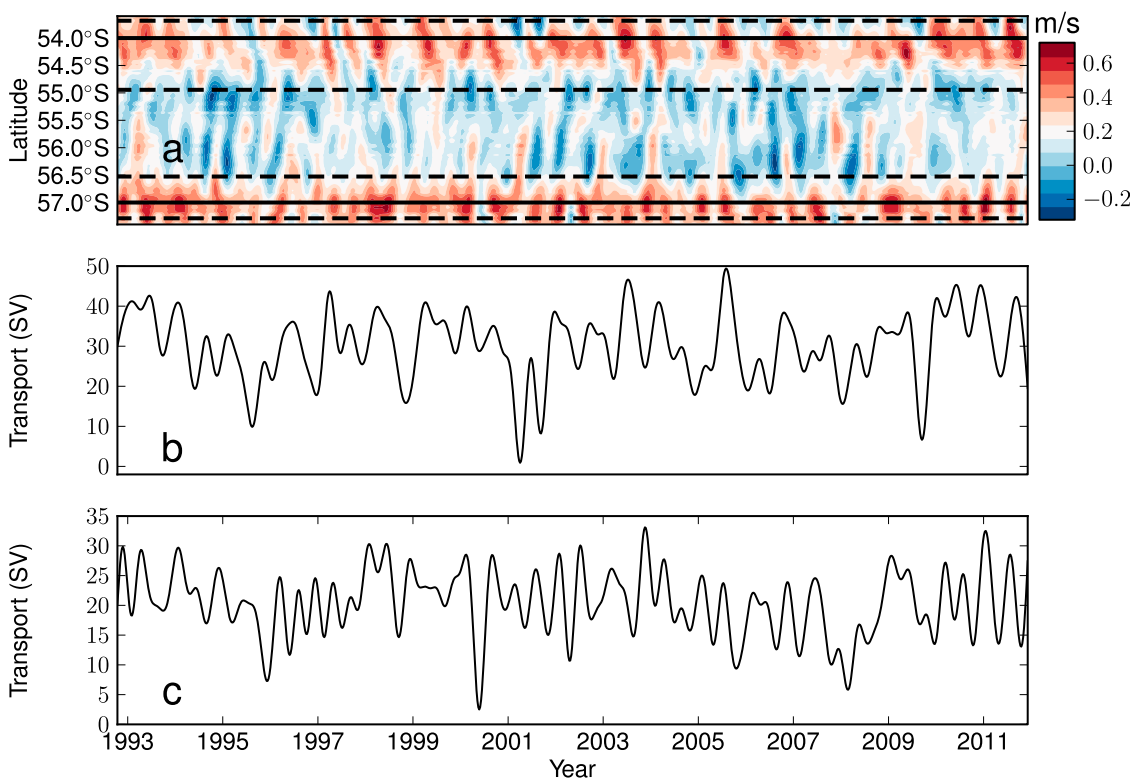

FIG. 11. Variability of the Southern Ocean near the Pacific-Antarctic Rise: (a) Latitude-time Hovmöller diagram of zonal velocity (thick black lines indicate approximate mean jet core position. Dashed lines indicate the northern and southern extents used in the transport computation): (b) transport of the northern jet and (c) transport of the southern jet.

The SSH anomaly field associated with key state A for each case study region is shown in Figs. 12a(i), 12b(i), and $12 \mathrm{c}(\mathrm{i})$. In each case, localized vortices are found on either side of the jets involved in the jet jumping variability. When the system is in state A (stronger southern jets), all cases show an anticyclonic vortex between the jets. The polarity of the vortices is reversed when the system is in state B [Figs. 12a(ii), 12b(ii), and 12c(ii)]. This situation is reminiscent of the vortex dipole that formed in the simulations of Chapman and Hogg (2013) and follows the schematic shown in Fig. 2.

As in Chapman and Hogg (2013), we compute the temporal variation of the strength of these vortices by the method described in section $3 \mathrm{~d}(3)$. The integration domain is determined by inspecting Fig. 12 to estimate the vortex extents. In each case, the integration domain $[\Omega$ in Eq. (2)] is indicated in Fig. 12. The time series of vortex strength is then compared to the PC time series that describes the jet jumping mode. This is shown for the three regions in Fig. 13 along with the square of the Pearson $R$ correlation coefficient. In the case of the Macquarie Ridge and the Pacific-Antarctic Rise, the time series show strong correlation with $R^{2}$ of 0.77 and 0.70 , respectively. The correlation is weaker at the Southeast Indian Ridge, with an $R^{2}$ of 0.46 . However, inspection of the time series shows periods of strong correlation (e.g., the period between 1994 and 1996), and periods of weaker correlation (e.g., between 2007 and 2008). We test the statistical significance of the correlation using a standard $p$ test and find that for the number of records in our data series (and assuming that each record is independent) the correlation is significant at the $95 \%$ level for both the Macquarie Ridge and the Pacific-Antarctic Rise. The correlation at the Southeast Indian Ridge is not significant at $95 \%$, but is significant at $90 \%$. As the flow in this region is less constrained by the topography, this reduced correlation is not surprising.

There are some notable differences in the spatial structure of the variability between the case studies. Chiefly, there is a difference downstream of the topographic features. At the Macquarie Ridge, there is evidence of downstream propagation of alternating positive and negative vorticity that broadly follows the path of the mean jet core position. This can be seen more clearly in Fig. 14, which shows a transect of anomalous SSH associated with key states A and B along the mean jet core position (shown in Fig. 6b). The oscillating positive/ negative SSH suggests a wavelike feature propagating along the jet core. Given that the ACC is known to act as a waveguide for Rossby waves (Hughes 1996), it is likely these are topographic Rossby waves that originate at the Macquarie Ridge and propagate along the jet downstream. Note that the downstream propagation is weaker than the upstream propagation, possibly due to the influence of the large Campbell Plateau to the east.

In contrast, there is no evidence for this phenomenon at either the Southeast Indian Rise or the PacificAntarctic Rise. Both of these topographic features are 
a) $\mathrm{i}$

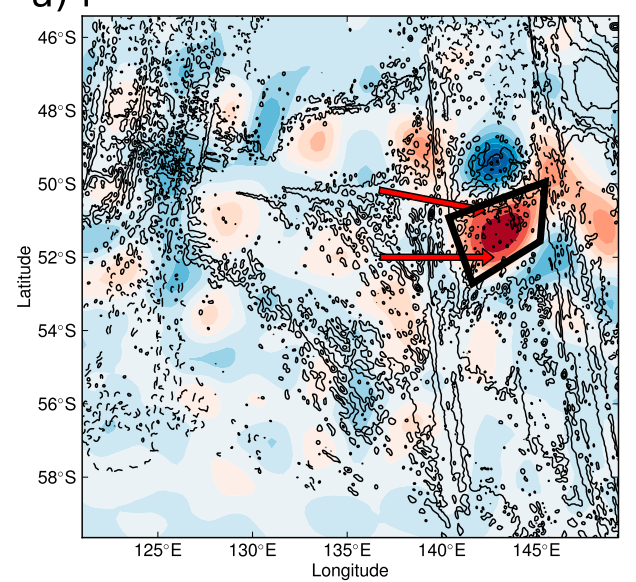

b) $\mathrm{i}$
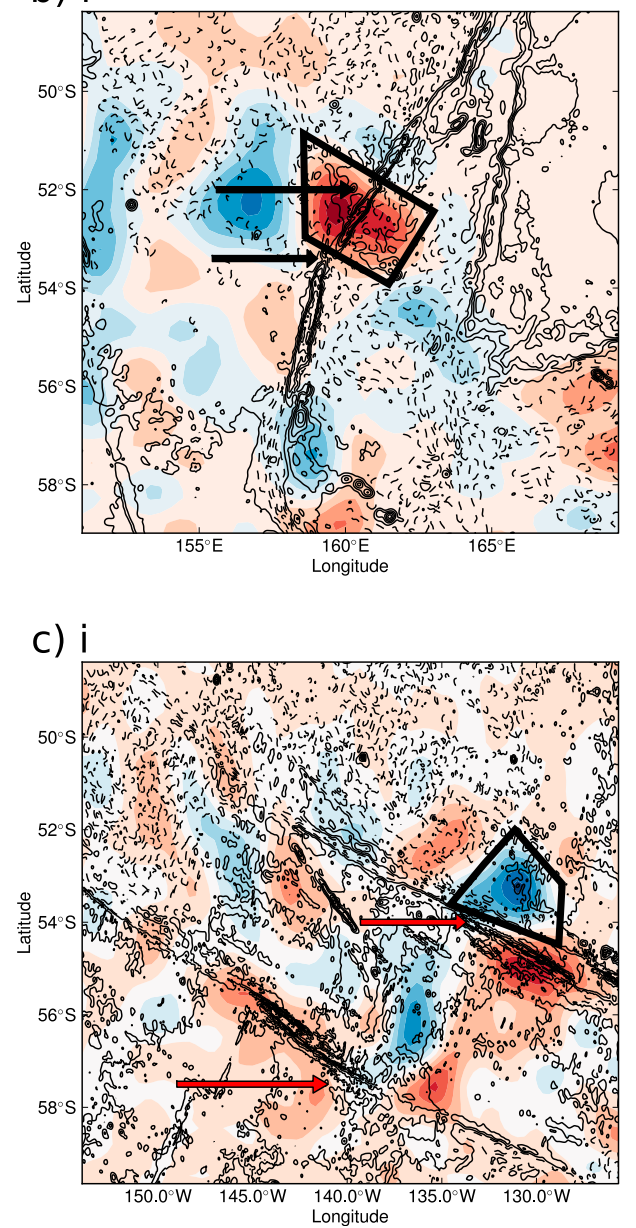

a) ii

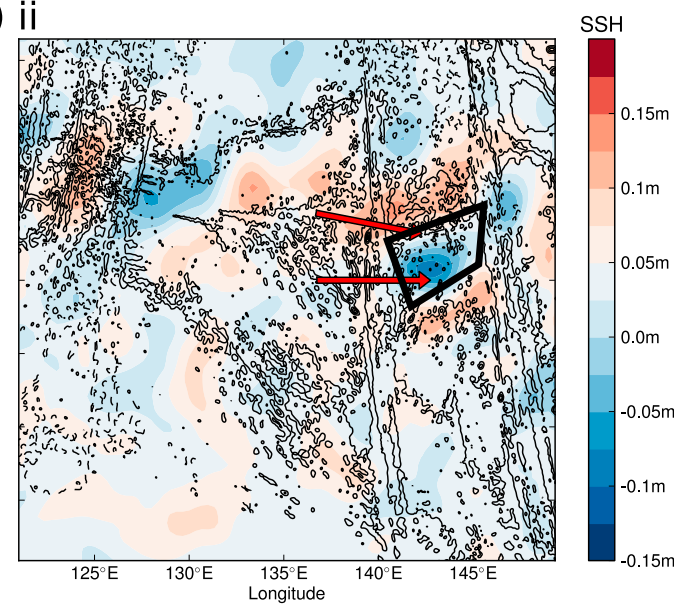

b) ii

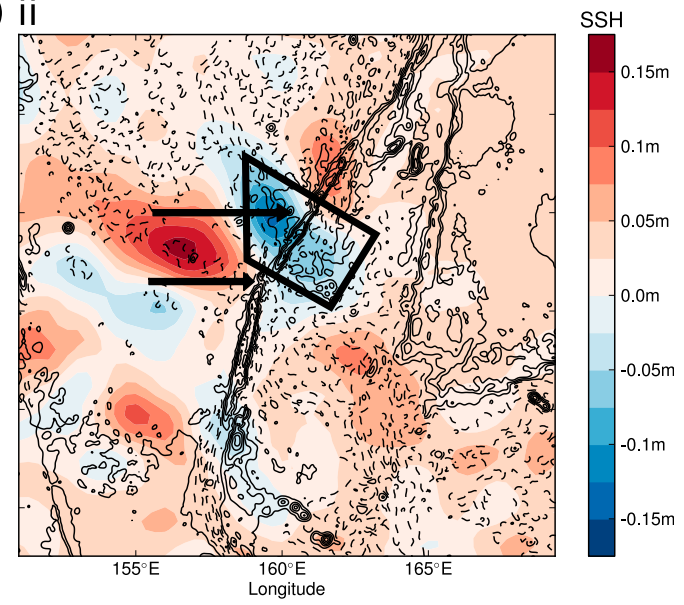

c) ii

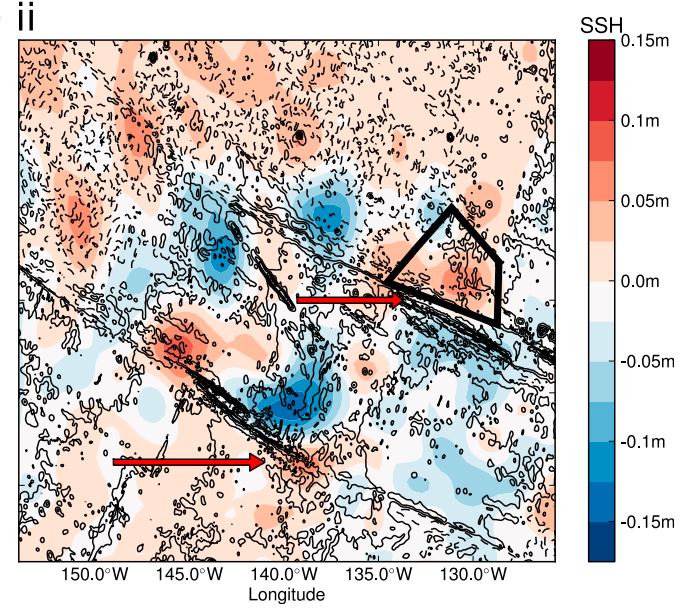

FIG. 12. Spatial patterns of SSH variability associated with key states of the jet jumping mode at each location: (i) key state A (stronger southern jet) and (ii) key state B (weaker southern jet) at the (a) Southeast Indian Ridge, (b) Macquarie Ridge, and (c) Pacific-Antarctic Rise. Arrows indicate approximate jet core positions. Solid black boxes indicate the integration domains $\Omega$ for vorticity computations. 

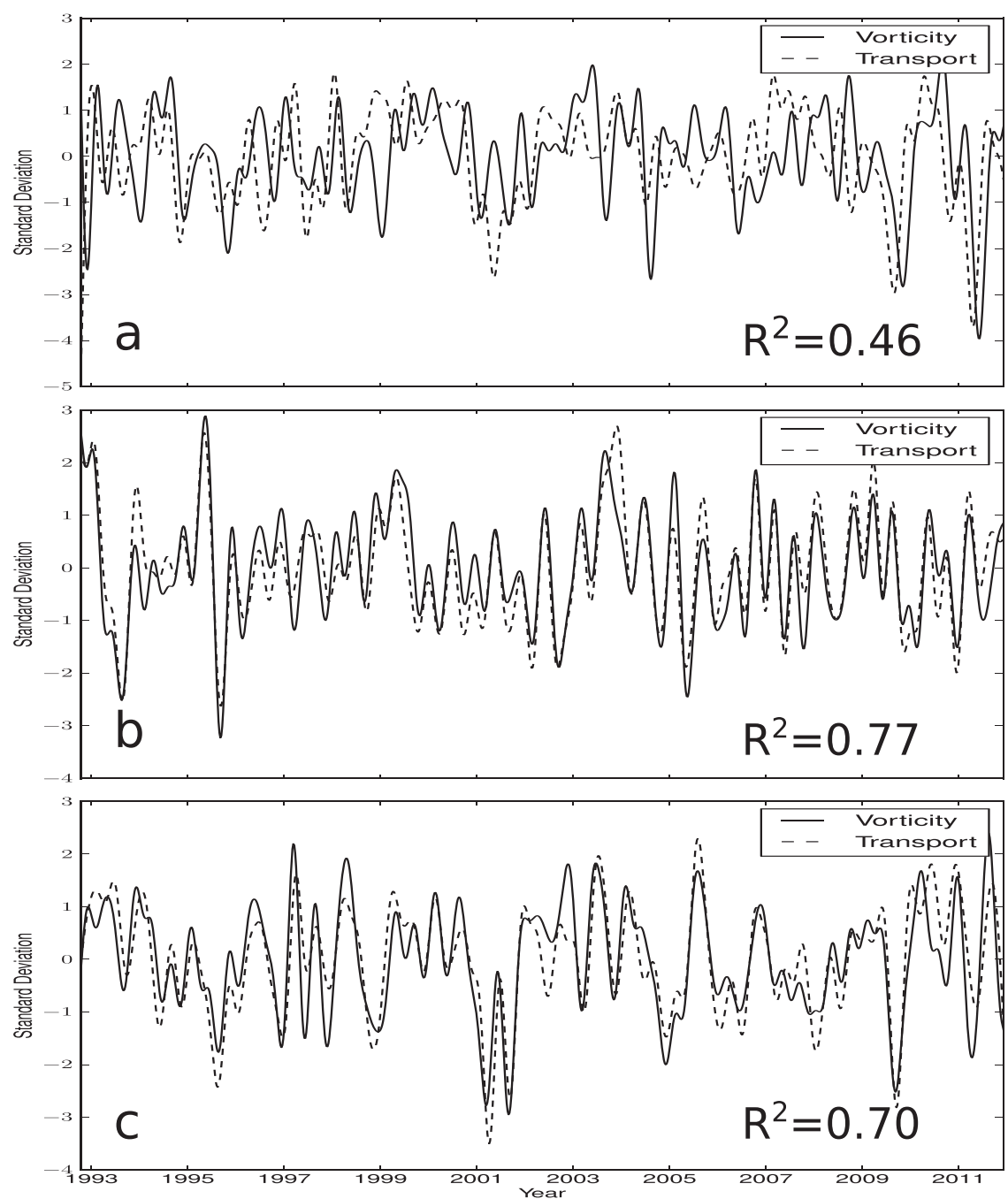

FIG. 13. Time series of the spatially averaged vorticity of the vortex indicated in Fig. 12 (solid) and transport (dashed) through the dominant canyon, both time series normalized by standard deviation: (a) Southeast Indian Ridge, (b) Macquarie Ridge, and (c) Pacific-Antarctic Rise. The $R^{2}$ values reported in each figure refer to the square of the Pearson $R$ correlation coefficient for the transport time series and the integrated vorticity time series for each case study.

significantly greater in zonal extent when compared to the Macquarie Ridge. Instead of a propagating feature, the topographic vortices are more localized to the region in between the two jets that undergo jet jumping.

Despite the complexity of the different topographic features, all three case studies presented exhibit some degree of jet jumping and topographic vortices with variable strength. Each case gives rise to characteristic vortex dipoles on either side of the variable jets. The principal component describing jet jumping variability is found to be highly correlated with the strength of these vortices. This analysis suggests that the hypothesis of Chapman and Hogg (2013) may be valid in the ocean.

\section{Comparison with numerical results}

To better understand the dynamics of jet jumping, we now discuss a series of numerical experiments extending those of Chapman and Hogg (2013). The configuration is essentially unchanged from that paper: using the ocean core of the Quasi-Geostrophic Coupled Model (Q-GCM) (Hogg et al. 2003), three-layer quasigeostrophic flow in a zonally reentrant $\beta$-plane channel is driven by a sinusoidal wind stress over a meridionally oriented ridge. The ridge contains two canyons that are the preferred pathways for the jets. We use a grid spacing of $5 \mathrm{~km}$, which ensures adequate representation of eddy physics for the chosen deformation radii $(\sim 15 \mathrm{~km})$. 


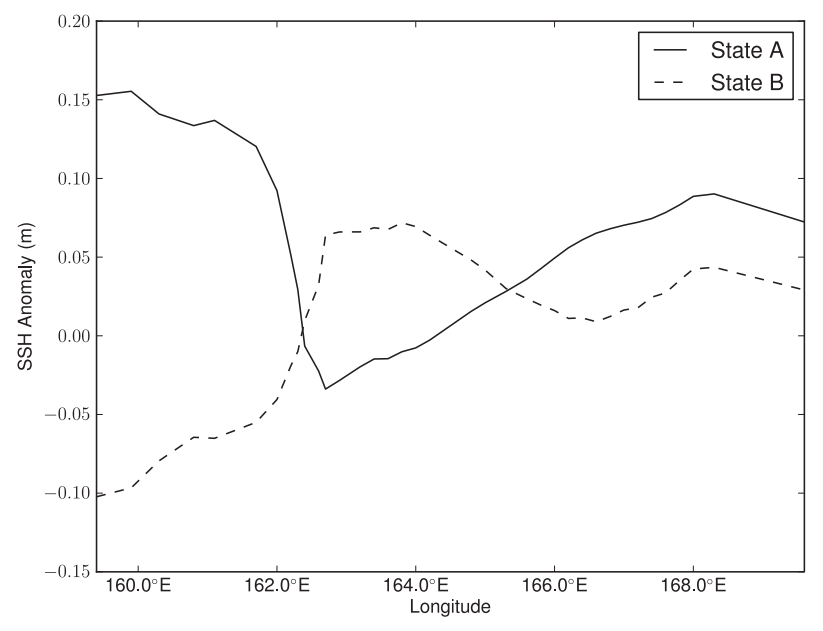

FIG. 14. Anomalous SSH variability for key state A (solid) and key state B (dashed) along the mean jet core position downstream of the Macquarie Ridge.

Details of the model are omitted for brevity, but may be found in Chapman and Hogg (2013).

The numerical experiments serve to complement the analysis performed using satellite altimetry. With the idealized configuration and well-known limitations of quasigeostrophic (QG) models (Williams et al. 2010; Thompson and Sallée 2012), these are not attempts to accurately model the flow. For this paper, the modeling has three purposes:

- show that internal variability is sufficient to generate jet jumping and that external forcing is not required,

- qualitatively compare the spatial structure of the jet jumping mode from the numerical model with that from the altimetry, and

- elucidate which parameters control the dynamics of the jet jumping variability.

Although the configuration of these experiments is essentially the same as in Chapman and Hogg (2013), there are several differences between that study and the numerical experiments described here. Chapman and Hogg was a process study; the results of a numerical simulation were used to provide support for their proposed dynamical framework. They did not explore the parameter space or the effect of changing the topography. In contrast, here we describe the effects of changes in the topographic shape and bottom friction on the jet jumping variability with the intention of qualitatively relating the numerical output to the results of section 4 , a task that is difficult to achieve with a single simulation given the differences between the case study flow fields and topographies. The partial exploration of the parameter space also serves to illuminate the physical reasons for any differences in the jet jumping variability observed in between case studies.
We principally investigate the effects of changing the topographic shape and changing the bottom friction. For the former, we set the topography as

$$
h(x, y)= \begin{cases}0, & x<-2 L_{\mathrm{hw}} \\ h_{0}(y) \cos ^{2}\left(\frac{\pi}{4} L_{\mathrm{hw}} x\right), & -2 L_{\mathrm{hw}}<x<2 L_{\mathrm{hw}} \\ 0, & y>2 L_{\mathrm{hw}},\end{cases}
$$

where $h_{0}(y)$ is the meridionally varying part of the topography that includes the canyons and $L_{\mathrm{hw}}$ is the topography half-width in the zonal direction. The $h_{0}(y)$ encapsulates the two canyons. These canyons have the profile

$$
h_{0}(y)=H_{0}\left\{1-\frac{1}{2} \cos ^{2}\left[\frac{\pi}{4} L_{c}\left(y-y^{\star}\right)\right]\right\},
$$

where $H_{0}$ is the maximum ridge height, $L_{c}$ is the halfwidth of the canyons, and $y^{\star}$ is the location of the canyon center. The two canyons are placed $500 \mathrm{~km}$ apart, each $250 \mathrm{~km}$ to the north and south of the meridional center of the domain (i.e., $y^{\star}=2250$ and $2750 \mathrm{~km}$ ). We set the canyon half-width to $L_{c}=100 \mathrm{~km}$.

We then run two sets of experiments where the hill half-width is changed. In the first set of experiments, the topography is "wide," with a half-width $L_{\mathrm{hw}}=1000 \mathrm{~km}$, which is designed to resemble features with greater zonal extent, such as the Pacific-Antarctic Rise. The second set uses a topographic feature that is "narrow," with a zonal half-width $L_{\mathrm{hw}}=200 \mathrm{~km}$. This topography is chosen to resemble smaller features such as the Macquarie Ridge.

The total ocean depth $H$ is set to $4000 \mathrm{~m}$. The layer thicknesses chosen are $300 \mathrm{~m}$ for the upper layer, $1100 \mathrm{~m}$ for the middle layer, and $2600 \mathrm{~m}$ for the lower layer. The topography height $H_{0}$ is set to $45 \%$ of the lower-layer depth. The topography is of sufficient depth to form regions of closed geostrophic contours $(f / H)$, which Chapman and Hogg (2013) found necessary for the manifestation of jet jumping variability.

Wind forcing is a zonally and temporally invariant field that has a single peaked sinusoidal profile in the meridional direction, identical to that of Chapman and Hogg (2013). We reiterate that there is no temporal variability in the wind field. Hence, all variability to manifest in the model is intrinsic.

We explicitly vary the bottom friction in order to change both the temporal variability of the topographic vortices [described in Chapman and Hogg (2013) and Venaille et al. (2011)] and the jet structure. Thompson and Young (2007) have shown that increasing the bottom 
friction results in more baroclinic jets that are more unstable to small perturbations, while Berloff et al. (2011) showed that bottom friction was the primary control on the strength of jets relative to the background eddy field. Increasing the bottom friction also tends to decrease the spacing between jets (Thompson 2010). As such, a variety of the changes in the structure of Southern Ocean jets can be induced in the numerical model by varying the bottom friction. In the Q-GCM, bottom friction is parameterized by a linear Rayleigh drag (Hogg et al. 2003). The drag is proportional to the depth of the bottom Ekman layer $E$ :

$$
\mathcal{F}=-E \nabla^{2} \psi,
$$

where $\psi$ is the geostrophic streamfunction. To evaluate the effect of changing bottom friction and the width of the topographic ridge, the numerical model is run using four different bottom Ekman layer depths, $2 \mathrm{~m}, 5 \mathrm{~m}$, $10 \mathrm{~m}$, and $20 \mathrm{~m}$, and two topographic configurations, wide and narrow, in a suite of eight experiments. Each individual simulation is spun up to a statistically steady state and then run for an additional 30 years, which returns enough data to extract meaningful statistics.

\section{a. Variability in the numerical model}

Time-mean upper-layer zonal velocity is shown in Fig. 15 for four different simulations. In each case we see multiple quasi-zonal jets that are steered by the topography and preferentially pass through the canyons, reminiscent of the ocean flows described in section 4. In both wide and narrow topographies, jets are steered through the canyons. We note that, as the bottom Ekman layer depth decreases, the surface velocity increases.

The output of each experiment is examined for evidence of jet jumping. We determine the spatial structure of the variability using the same thresholding technique described in section 3 and employed on the satellite altimetry data in section 4 . The key states for each individual simulation are shown in Fig. 16. In each case, we find the appearance of either a vortex between the northern and southern canyons or a vortex dipole with one pole on either side of the northern canyon. As in Chapman and Hogg (2013), the strength of these vortices (as measured by the spatially integrated relative vorticity) is highly temporally variable and strongly correlated with the PC time series of the jet jumping mode. Closer investigations have revealed that, even in cases where it appears that there is only a vortex monopole, vortex dipoles exist, although the northern vortex is substantially weaker than the southern vortex. As the bottom Ekman layer depth increases, the strength of the dominant topographic vortex decreases [a result consistent with Dewar (1998), who studied the formation of similar topographic vortices]. However, the strength of the northern vortex relative to the southern vortex generally increases as the bottom Ekman layer depth increases. When $E=2 \mathrm{~m}$ (shown in Fig. 16a), the southern vortex is dominant. When $E=20 \mathrm{~m}$ (Fig. 16d), the strengths of the two vortices are almost equal.

Despite its simplicity, the QG model has managed to qualitatively reproduce the spatial structure of the observed jet jumping mode. The numerical model shows some quantitative agreement with the observations. This can be seen in Table 1, which compares the mean and standard deviations of the transport through the dominant pathway (i.e., the pathway that carries the higher mean transport). The numerical experiments generally have higher transports and standard deviations than those found in the altimetric analysis. This is unsurprising given the greater total depth in the model $^{1}$ and the lack of upstream topography to decelerate the jet flows. However, when one examines the relative standard deviation (as a percentage of total transport), the model, while having a low bias, gives results with a similar order of magnitude. Given the idealized nature of the simulations, this level of agreement suggests that the model is capturing at least some of the physics occurring in the ocean. We also reiterate that, since the external forcing applied was time invariant, the jet jumping variability can arise through internal variability alone. However, this does not imply that time-variable wind forcing may not affect the variability of the jets near topography.

There is a difference in this structure between the wide and narrow topographies. In simulations with narrow topography, there is evidence of downstream propagation of the topographic vortices. This can be seen most clearly between $x=5000 \mathrm{~m}$ and $x=5500 \mathrm{~km}$ in Figs. 16e, 16g, and 16h. The propagation downstream can extend for several wavelengths before dissipating. In contrast, there is limited evidence of downstream propagation of the topographic vortex in simulations with wide topography. In these cases, the topographic vortex is more strongly confined to the topography.

Comparing this difference in spatial structure between wide and narrow cases to the observational case studies, we can see qualitative similarities. As discussed in section $4 \mathrm{~d}$, at the Macquarie Ridge vortex features show downstream propagation along the jet core (shown in Figs. 12b and 14), while at the Pacific-Antarctic Rise the topographic vortex is more strongly confined to the

\footnotetext{
${ }^{1}$ Owing to constraints of the QG approximation, topography must be small relative to total depth.
} 


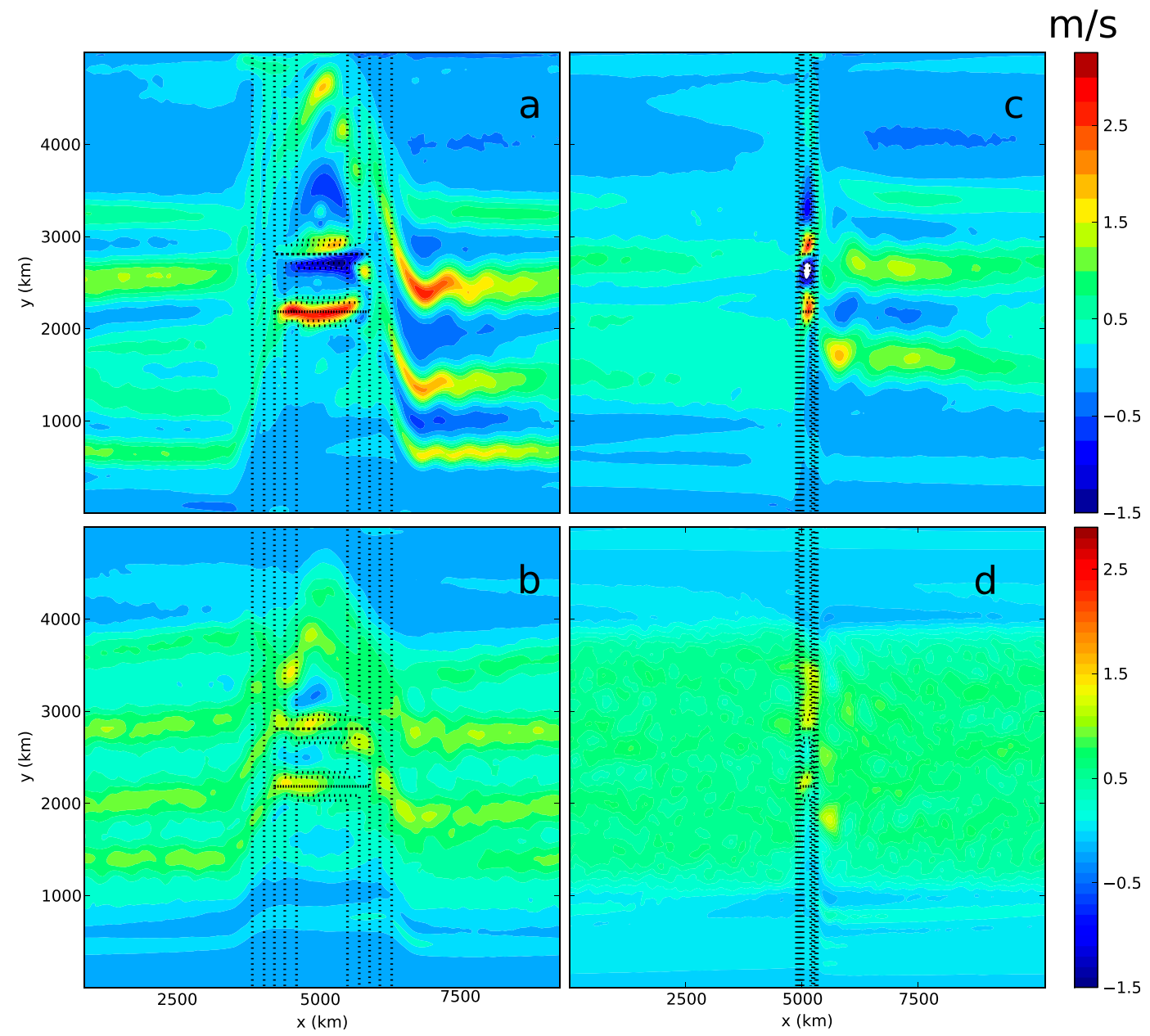

FIG. 15. Time-mean upper-layer geostrophic zonal velocity $\left(\mathrm{m} \mathrm{s}^{-1}\right)$ in the numerical model for four ensemble members: (a) wide topography, Ekman layer depth of $2 \mathrm{~m}$; (b) wide topography, Ekman layer depth of $20 \mathrm{~m}$; (c) narrow topography, Ekman layer depth of $2 \mathrm{~m}$; and (d) narrow topography, Ekman layer depth of $20 \mathrm{~m}$. Dotted lines indicate depth contours (contour spacing of $250 \mathrm{~m}$ ).

ridge separating the two jets (Fig. 12c). The qualitative similarities described here allow us to posit that the difference in structure is induced by the differences in the shape of the topography.

As alluded to above, cases showing downstream propagation of vortices hint at possible differences in the underlying dynamics: possibly dominated by Zapiolalike circulations (Volkov and Fu 2008) in the wide topography cases and becoming more dominated by standing Rossby waves in the latter. This may have implications for jet jumping variability, as the time scales and persistence of these features may be controlled by different dynamics.

\section{b. Control of the jet jumping time scale}

The study of the Southern Ocean regions showed differing time scales of variability. Are the differences between case studies related to the topographic geometry or to changes induced by varying the bottom friction? To answer this question, we compare the time scale of variability that arises between the different ensemble members.

To analyze the variation in time scale, we compute the spectra of the time series transport through the southern canyon and the principal component time series of the jet jumping mode. We define the "dominant period" as the period of the largest magnitude spectral peak present in both time series. The dominant period for each ensemble member is shown in Fig. 17.

Figure 17 clearly shows the influence of the changing bottom Ekman layer depth on the time scale of the jet jumping mode: as the bottom friction increases, the dominant period becomes shorter. In situations with deeper bottom layers the jet jumping time scale shifts to 

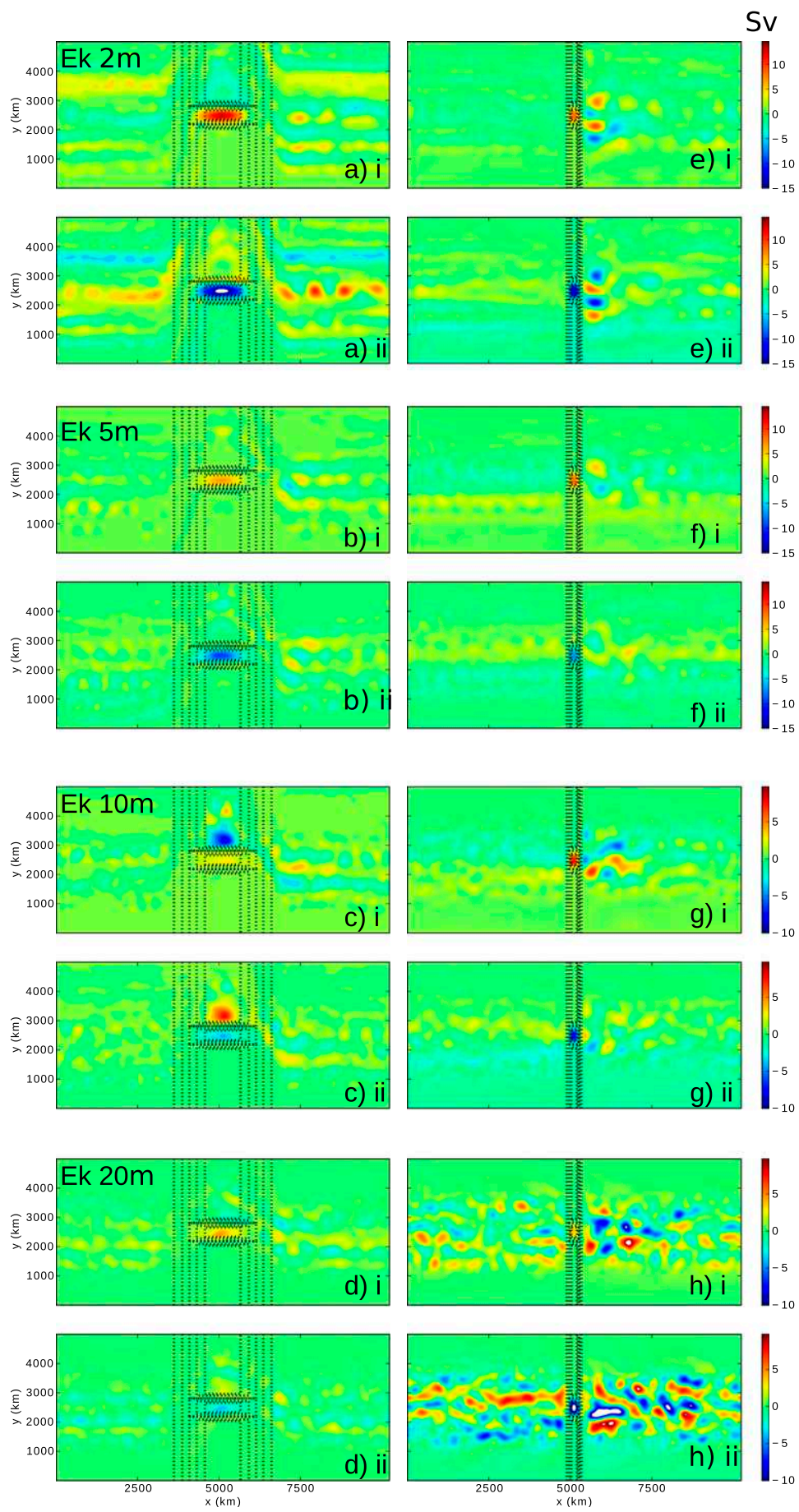

FIG. 16. Spatial patterns of streamfunction (Sv) variability in the lower layer associated with key states of the jet jumping mode for all members of numerical ensemble: (i) key state A (anomalously high transport in the southern canyon) and (ii) key state B (anomalously high transport in the northern canyon). (a) Wide topography, Ekman layer depth of $2 \mathrm{~m}$; (b) wide topography, Ekman layer depth of $5 \mathrm{~m}$; (c) wide topography, Ekman layer depth of $10 \mathrm{~m}$; (d) wide topography, Ekman layer depth of $20 \mathrm{~m}$; (e) narrow topography, Ekman layer depth of $2 \mathrm{~m}$; (f) narrow topography, Ekman layer depth of $5 \mathrm{~m}$; (g) narrow topography, Ekman layer depth of $10 \mathrm{~m}$; (h) narrow topography, Ekman layer depth of $20 \mathrm{~m}$. Dotted lines indicate depth contours (contour spacing of $250 \mathrm{~m}$ ) Note the change in colormap scale between the cases with Ekman layer depths of (top) 2 and $5 \mathrm{~m}$ and cases with Ekman layer depths of (bottom) 10 and $20 \mathrm{~m}$. 
TABLE 1. Comparison of mean and variability of transport between the ocean case studies and the numerical model.

\begin{tabular}{lccc}
\hline \multicolumn{1}{c}{ Location } & Transport (Sv) & $\sigma(\mathrm{Sv})$ & $\sigma(\%$ of mean transport) \\
\hline Southeast Indian Ridge & 27.3 & 8.2 & $30.0 \%$ \\
Macquarie Ridge & 39.2 & 5.0 & $12.8 \%$ \\
Pacific-Antarctic Rise & 30.2 & 8.4 & $27.7 \%$ \\
Wide topography, Ekman depth 2m & 216.7 & 30.0 & $13.8 \%$ \\
Wide topography, Ekman depth 5 m & 136.4 & 15.0 & $11.0 \%$ \\
Wide topography, Ekman depth 10 m & 94.2 & 16.2 & $17.2 \%$ \\
Wide topography, Ekman depth 20 m & 76.0 & 22.9 & $30.0 \%$ \\
Narrow topography, Ekman depth 2m & 221.6 & 20.7 & $9.34 \%$ \\
Narrow topography, Ekman depth 5 m & 114.4 & 19.4 & $17.0 \%$ \\
Narrow topography, Ekman depth 10 m & 62.7 & 19.2 & $30.6 \%$ \\
Narrow topography, Ekman depth 20 m & 56.7 & 14.6 & $25.7 \%$ \\
\hline
\end{tabular}

higher frequencies. This is consistent with the conclusions of Chapman and Hogg (2013) and Venaille et al. (2011), who suggested that the time scale of the topographically generated vortices scaled inversely with the bottom friction. We also observe that the wider topography induces jet jumping with a longer period than the narrow topography.

Making a simple comparison of the time scales obtained from the numerical modeling to those found in the observational case studies, the calculated dominant period for the jet jumping variability at the narrow Macquarie Ridge $(\sim 2.2 \mathrm{yr})$ is $\sim 31 \%$ lower than that calculated at the wider Pacific-Antarctic Rise $(\sim 3.2 \mathrm{yr})$. The time scales computed from the numerical experiments show a similar reduction in time scale between the wide and narrow cases, seen in Fig. 17. With an Ekman depth of $2 \mathrm{~m}$, the dominant jet jumping period with narrow topography reduces by $21 \%$ when compared to the wide topography. With an Ekman depth of $5 \mathrm{~m}$, the reduction in time scale is $25 \%$.

The fact that the differences in jet jumping time scales observed between the Macquarie Ridge and PacificAntarctic Rise can be induced in the model by changing the shape of the topography suggests that the observed differences may be attributable (at least in part) to the differences in the shape of the topography at the two case study sites. As mentioned in section $5 b$, the spatial structure of jet jumping variability near narrow topography appears to be dominated more by standing Rossby waves, while near wide topography it is dominated by Zapiola-like circulations. The differences in dynamical origin of the topographic vortices may explain the differences in observed time scales.

\section{Discussion and conclusions}

In this paper, we have studied a form of low-frequency variability called "jet jumping" using both AVISO satellite altimetry and a series of simulations from an idealized quasigeostrophic numerical model. Jet jumping variability manifests itself as an anticorrelated strengthening and weakening of adjacent jets that pass near the same topographic feature. Choosing three regions in the Southern Ocean as case studies, we demonstrate that the jet jumping behavior exists and explains a substantial portion of the transport variability at each region. Using the key state analysis described by Berloff et al. (2007), we determine the spatial structure of the variability and find a degree of similarity in each region. In each case, a vortex dipole aligned with the topography forms between the two jet paths. This provides some observational support for the mechanism proposed in Chapman and Hogg (2013). The analysis indicates wavelike propagation of these vortex features at the narrow Macquarie Ridge and Southeast

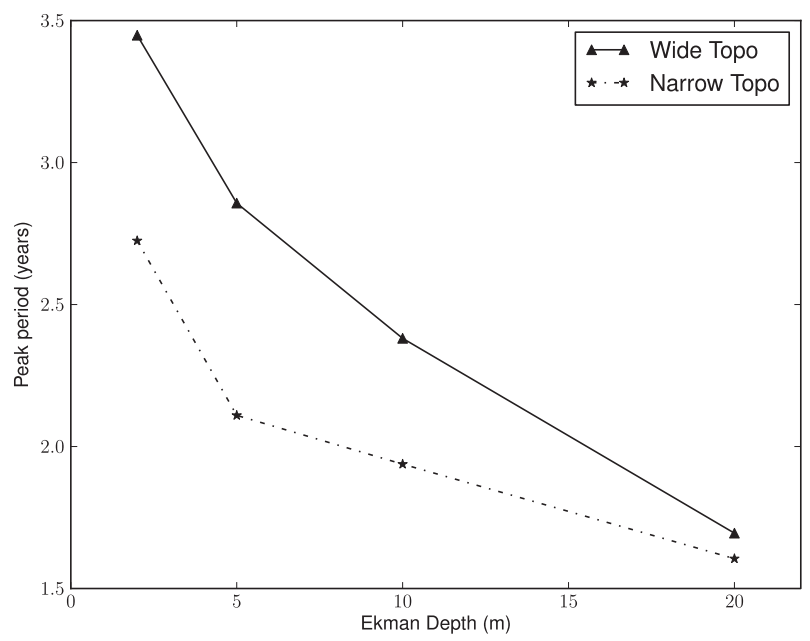

FIG. 17. Dominant spectral peak of the principal component associated with the jet jumping mode for the wide (solid triangles) and narrow (dashed stars) topography at varying Ekman numbers. 
Indian Rise, while the vortices are fixed to topography at the wider Pacific-Antarctic Ridge.

A comparison between the strength of the topographic vortices (measured by the spatially averaged relative vorticity) and the transport time series shows a high level of correlation in all three case studies. This lends support to the hypothesis proposed in Chapman and Hogg that the variability is primarily controlled by temporal variability in the strength of the vortices, which are themselves driven by turbulent eddy fluxes or wave activity.

We have used a series of numerical experiments to further investigate this mode of variability. Using idealized topography based on the experiments of Chapman and Hogg (2013) we are able to induce jet jumping variability. Using the same key state analysis that was applied to the altimeter fields, we obtain a spatial structure that is qualitatively similar to those found in the Southern Ocean regions. The model also shows a quantitative similarity with the results obtained from the altimetry.

Using the suite of simulations, we can judge the effect of changing the topographic length scale and bottom friction on the manifestation of the variability. Topographies with smaller zonal length scales appear to decrease the dominant time scale of the variability, while increasing bottom friction also acts to decrease the jet jumping time scale.

There are some discrepancies between the modeling and observations. In particular, while the key state analysis of the numerical output shows the formation of a vortex dipole, consistent with the observations, there is an asymmetry present that is not present in the observational studies. The mean area-averaged relative vorticity of the southern pole is typically much higher than the same metric computed for the northern pole. This asymmetry is more pronounced at smaller values for the Ekman depth. Only at the Macquarie Ridge is such an asymmetry present, and it is substantially weaker than in the simulations.

If the dynamical mechanism proposed in Chapman and Hogg (2013) is correct, this false asymmetry may lead to changes in magnitude of the variability as the transport through the canyons is related to the difference of relative vorticity between the two topographic circulations. This may impact our ability to accurately model the dynamics of the flow in this framework.

In addition, we have not investigated the effects of external forcing on this mode of variability. It is conceivable that wind, by generating local changes in eddy kinetic energy, may have a role to play in inducing variability of jets near topography. In addition, we have not explored the full parameter space to determine what effects changing other parameters, such as stratification or the orientation of topography, would have on the variability. Further work could also attempt to model the variability accurately using a more realistic model.

Despite these shortcomings, this study has shown that the jet jumping variability exists in various places in the ocean and can be studied with altimetric data. It has also demonstrated the applicability of the Chapman and Hogg (2013) framework for understanding the dynamics and why such transitions take place.

Acknowledgments. The authors would like to acknowledge Drs. A. McC. Hogg and S. R. Rintoul for helpful discussions along with two anonymous reviewers whose comments greatly improved the manuscript. The altimeter products were produced by SSALTO/DUACS and distributed by AVISO with support from CNES. Numerical computations were supported by an award under the Merit Allocation Scheme on the National Facility of the Australian Partnership for Advanced Computing. C. C. Chapman is supported by a CSIRO Wealth from Oceans Flagship Scholarship.

\section{APPENDIX}

\section{Computation of Vorticity in Mercator Coordinates}

Satellite altimetry fields are provided on a Mercator grid at fixed latitude/longitude intervals. We write latitude as $\varphi$ and longitude as $\lambda$ and the respective grid spacing as $\Delta \varphi$ and $\Delta \lambda$. In a Mercator grid system on a spherical surface with radius $R_{E}$, the distance along a line of constant latitude $d x$ and along a line of constant longitude $d y$ is defined by the anholonomic transform

$$
\begin{aligned}
& d x=R_{E} \cos \varphi d \lambda \quad \text { and } \\
& d y=R_{E} d \varphi
\end{aligned}
$$

which gives the covariant metric components

$$
g_{11}=R_{E}^{2} \cos ^{2} \varphi ; \quad g_{12}=0 ; \quad g_{21}=0 ; \quad \text { and } \quad g_{22}=R_{E}^{2} .
$$

In this paper, we compute vorticity from SSH [as in Eq. (2)], which would require use of the Laplace operator in Cartesian coordinates. In Mercator coordinates, we use the generalized Laplace-Beltrami operator:

$$
\tilde{\nabla}^{2} \psi=\frac{1}{\sqrt{|g|}} \frac{\partial}{\partial \tilde{x}^{i}}\left(\sqrt{|g|} g^{i j} \frac{\partial}{\partial \tilde{x}^{j}} \psi\right),
$$

where $\tilde{x}^{1}=\varphi$ and $\tilde{x}^{2}=\lambda$, and $g^{i j}$ are the components of the contravariant metric, which is determined from the previously defined covariant metric by $g_{i j} g^{i j}=\delta_{i}^{j}$. 


\section{REFERENCES}

Amante, C., and B. W. Eakins, 2009: Etopo1 1 arc-minute global relief model: Procedures, data sources and analysis. NOAA Tech. Memo. NESDIS NGDC-24, 19 pp. [Available online at http://www.ngdc.noaa.gov/mgg/global/relief/ETOPO1/docs/ ETOPO1.pdf.]

Arbic, B. K., R. B. Scott, D. B. Chelton, J. G. Richman, and J. F. Shriver, 2012: Effects of stencil width on surface ocean geostrophic velocity and vorticity estimation from gridded satellite altimeter data. J. Geophys. Res., 117, C03029, doi:10.1029/ 2011JC007367.

Berloff, P., A. M. C. Hogg, and W. Dewar, 2007: The turbulent oscillator: A mechanism of low-frequency variability of the wind-driven ocean gyres. J. Phys. Oceanogr., 37, 2363-2386.

— - S. Karabasov, J. T. Farrar, and I. Kamenkovich, 2011: On latency of multiple zonal jets in the oceans. J. Fluid Mech., 686, 534-567, doi:10.1017/jfm.2011.345.

Chapman, C. C., and A. M. Hogg, 2013: Jet jumping: Lowfrequency variability in the Southern Ocean. J. Phys. Oceanogr., 43, 990-1003.

Dewar, W. K., 1998: Topography and barotropic transport control by bottom friction. J. Mar. Res., 56, 295-328.

Dibarboure, G., M.-I. Pujol, F. Briol, P. Y. L. Traon, G. Larnicol, N. Picot, F. Mertz, and M. Ablain, 2011: Jason-2 in DUACS: Updated system description, first tandem results and impact on processing and products. Mar. Geod., 34, 214-241, doi:10.1080/01490419.2011.584826.

Graham, R. M., A. M. de Boer, K. J. Heywood, M. Chapman, and D. P. Stevens, 2012: Southern Ocean fronts: Controlled by wind or topography? J. Geophys. Res., 117, C08018, doi:10.1029/2012JC007887.

Hogg, A. M., W. K. Dewar, P. D. Killworth, and J. R. Blundell, 2003: A quasi-geostrophic coupled model (Q-GCM). Mon. Wea. Rev., 131, 2261-2278.

Hughes, C. W., 1996: The Antarctic Circumpolar Current as a waveguide for Rossby waves. J. Phys. Oceanogr., 26, 13751387.

— , and E. R. Ash, 2001: Eddy forcing of the mean flow in the Southern Ocean. J. Geophys. Res., 106 (C2), 2713-2722.

Marshall, D. P., 2011: Rossby wormholes. J. Mar. Res., 69, 309-330.

Orsi, A. H., T. I. Whitworth, and W. D. J. Nowlin, 1995: On the meridional extent and fronts of the Antarctic Circumpolar Current. Deep-Sea Res. I, 42, 641-673.

Phillips, H., 2000: Mean flow, eddy variability and energetics of the subantarctic front south of Australia. Ph.D. thesis, University of Tasmania, $158 \mathrm{pp}$.

Rhines, P. B., 1994: Jets. Chaos, 4, 313-339, doi:10.1063/1.166011.

Rintoul, S. R., C. W. Hughes, and D. Olbers, 2001: The Antarctic Circumpolar Current system. Ocean Circulation and Climate, G. Siedler et al., Eds., Academic Press, 271-302.
Rio, M. H., S. Guinehut, and G. Larnicol, 2011: New CNES-CLS09 global mean dynamic topography computed from the combination of grace data, altimetry, and in situ measurements. J. Geophys. Res., 116, C07018, doi:10.1029/2010JC006505.

Smith, S. W., 2003: The Scientist and Engineer's Guide to Digital Signal Processing. 2nd ed. California Technical Publishing, $640 \mathrm{pp}$.

Sokolov, S., and S. R. Rintoul, 2007: Multiple jets of the Antarctic Circumpolar Current south of Australia. J. Phys. Oceanogr., 37, 1394-1412.

, and 2009: Circumpolar structure and distribution of the Antarctic Circumpolar Current fronts: 2. Variability and relationship to sea surface height. J. Geophys. Res., 114, C11019, doi:10.1029/2008JC005248.

Stern, M. E., and G. R. Flierl, 1987: On the interaction of a vortex with a shear flow. J. Geophys. Res., 92 (C10), 10733-10 744.

Thompson, A. F., 2008: The atmospheric ocean: Eddies and jets in the Antarctic Circumpolar Current. Philos. Trans. Roy. Soc., A366, 4529-4541, doi:10.1098/rsta.2008.0196.

2010: Jet formation and evolution in baroclinic turbulence with simple topography. J. Phys. Oceanogr., 40, 257-278. , and W. R. Young, 2007: Two-layer baroclinic eddy heat fluxes: Zonal flows and energy balance. J. Atmos. Sci., 64, 3214-3231.

— , and K. J. Richards, 2011: Low frequency variability of Southern Ocean jets. J. Geophys. Res., 116, C09022, doi:10.1029/ 2010JC006749.

—_, and J.-B. Sallée, 2012: Jets and topography: Jet transitions and the impact on transport in the Antarctic Circumpolar Current. J. Phys. Oceanogr., 42, 956-972.

Venaille, A., J. Le Sommer, J. M. Molines, and B. Barnier, 2011: Stochastic variability of oceanic flows above topography anomalies. Geophys. Res. Lett., 38, L16611, doi:10.1029/2011GL048401.

Völker, C., 1999: Momentum balance in zonal flows and resonance of baroclinic Rossby waves. J. Phys. Oceanogr., 29, $1666-1681$.

Volkov, D. L., and L.-L. Fu, 2008: The role of vorticity fluxes in the dynamics of the Zapiola anticyclone. J. Geophys. Res., 113, C11015, doi:10.1029/2008JC004841.

Ward, M. L., and A. M. Hogg, 2011: Establishment of momentum balance by form stress in a wind-driven channel. Ocean Modell., 40, 133-146.

Wilks, D. S., 2006: Statistical Methods in the Atmospheric Sciences. 2nd ed. Academic Press, 627 pp.

Williams, P. D., P. L. Read, and T. W. N. Haine, 2010: Testing the limits of quasi-geostrophic theory: Application to observed laboratory flows outside the quasi-geostrophic regime. J. Fluid Mech., 649, 187-203.

Williams, R. G., C. Wilson, and C. W. Hughes, 2007: Ocean and atmosphere storm tracks: The role of eddy vorticity forcing. J. Phys. Oceanogr., 37, 2267-2289. 